\title{
LE NOYAU ET LE FONDEMENT
}

\section{FRANCIS WOLFF, LECTEUR DE METAPHYSIQUE Z}

Jorge MiTTELMANN

Universidad de los Andes (Chile)

Dans une étude dédiée au livre $\mathrm{Z}$ de la Métaphysique d'Aristote ${ }^{1}$, Francis Wolff avança une hypothèse novatrice, qui allait à l'encontre de l'opinion reçue selon laquelle les critères successifs qu'Aristote énonce au cours de ce livre débouchent sur deux réponses incompatibles à la question de savoir qu'elle est l'ousia : soit on se laisse guider par la recherche d'un sujet qui n'est jamais prédicat, en « déshabillant » chaque réalité sensible jusqu'à y trouver une pure matière ; soit on se tourne plutôt vers le noyau définissable que chaque réalité porte en elle-même, pour mettre à nu son essence comme réponse logique $(\lambda o \gamma(\kappa \tilde{\omega} \varsigma)$ à la question « qu'estce que c'est». Ces deux orientations, censées être « exclusives », recoupent la double démarche historique attestée déjà dans le livre $\mathrm{A}$, où le clivage opposait les naturalistes, épris du substrat, aux socratiques et platoniciens, qui cherchent l'immuable non pas dans la matière, mais dans les formes et quiddités définissables.

En s'appuyant sur la référence grammaticalement ambigu' du pronom

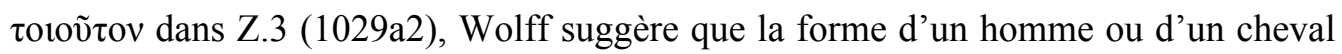
a, elle aussi, le droit d'être appelé « substrat », au même titre que sa matière et que le composé lui-même. Nous offrirons dans ce travail quelques confirmations textuelles supplémentaires de cette lecture, à l'aide de passages tirés surtout de la philosophie naturelle d'Aristote, qui semblent étayer l'attribution à la forme de la condition de « substrat ».

${ }^{1}$ «Qu'est-ce que se demander ce qu'est la réalité ? (Perspectives sur le livre Zêta de la Métaphysique) », dans Narcy, M. et Tordesillas, A. : La Métaphysique d'Aristote, Perspectives Contemporaines, Paris - Bruxelles, Vrin, 2005, p. 145-169. 


\section{Un seul critère, deux applications}

Dans Métaphysique Z.3, Aristote entreprend sa recherche sur la substance muni d'un critère " générale et schématique $»^{2}$ de réalité, dont il ne se départira pas tout au long du livre Z. D'après ce critère hérité des Catégories, est réel au sens propre et premier du terme «ce dont les autres choses sont dites, alors que lui-même n'est plus dit d'autre chose » (1028b36-37). Au gré de son application (tantôt logique, tantôt physique), le critère est censé mettre à nu aussi bien une réalité sous-jacente au discours et qui opère comme sujet premier d'attribution, qu'une réalité (elle-même inchangée) sous-jacente au changement. À côté de cet outil de recherche, on trouve regroupés dans un même ensemble les dispositifs permettant de répondre, à des degrés de précision variables, à la question socratique «qu'est-ce que...? » - question qui porte de préférence ${ }^{3}$ sur un substrat préalablement identifié par recours au premier critère ( $c f . \mathrm{Z}, 1,1028 \mathrm{a} 34-\mathrm{b} 1)$. Ces voies alternatives menant à l'ousia sont l'universel, le genre et la quiddité qui, précisément parce qu'elles dévoilent l'essence d'un substrat «qui n'est plus dit d'autre chose », attestent la primauté ontologique de ce dernier et la place de choix qui lui revient dans l'examen de la substance :

C'est pourquoi c'est d'abord lui [sc. le substrat] qu'il faut déterminer : car le substrat premier

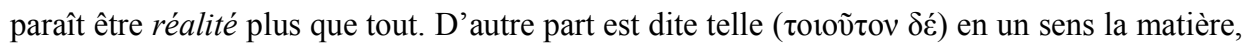
en un autre sens la forme, en un troisième sens le $<$ composé $>$ des deux (j'entends par matière par exemple le bronze, par forme la figure visible, et par <composé $>$ des deux la statue dans son ensemble). (1028b37-1029a5. Traduction Wolff).

Le sens de ce texte à double tranchant tourne pour une grande partie autour de la

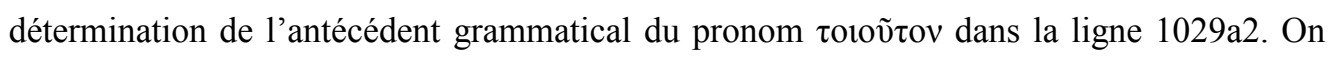
hésite entre (a) « substrat premier » (ou « substrat»), qui sont (dans le texte grec) les mots qui précèdent immédiatement celui dont la référence reste ambiguë ; et (b) l'expression « ousia », qu'on a rendue ici par « réalité ». Sous cette dernière interprétation, les trois items qu'Aristote dénombre - forme, matière et composé - s'ajoutent aux quatre acceptions du mot « réalité » qu'il a listées en début de chapitre : sujet, quiddité, genre et universel. De

\footnotetext{
${ }^{2}$ F. Wolff, «Qu'est-ce que se demander ce qu'est la réalité ? ... », art. cit., p. 153.

${ }^{3}$ Mais pas exclusivement : cf. Top. I, 9, 103b27-39; Z, 4, 1030b4-13.
} 
sorte que (comme le remarque Francis Wolff) «[1]es quatre candidats logiques à la substantialité ... se trouveraient alors en concurrence avec trois candidats physiques », ce qui pose la question du rapport problématique «entre les deux types de caractérisation de l'ousia $»^{4}$. Mais si l'on essaie de parer à cette inflation de prétendants par un autre choix grammatical, qui se porterait sur l'expression « substrat premier », les difficultés ne seront pas moindres. Notamment, on prêtera à Aristote la thèse que la matière et le composé mais aussi sa forme - «font substrat $»^{5}$ pour d'autres choses et peuvent satisfaire à la description d'une réalité sous-jacente « qui n'est plus dite d'autre chose ».

Or ce résultat est on ne peut plus déroutant (« immensely puzzling $)^{6}$, étant donné la présence avérée chez Aristote de la forme comme prédicat de la matière constitutive du composé : l'appartenance de ce dernier à une certaine classe naturelle à l'exclusion de toutes les autres tient à ce lien hylémorphique, dont l'expression prédicative rend claire la

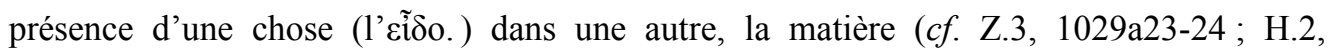

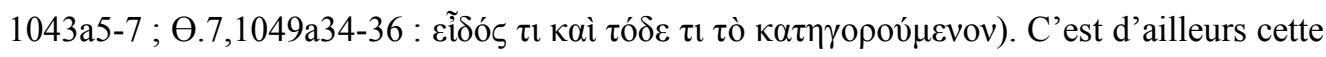
complexion prédicative interne qui risque de faire « imploser » la simplicité logique de ces sujets premiers d'attribution qui étaient reconnus un peu partout dans l'Organon, et dont Socrate et Bucéphale faisaient figure de porte-étendard: ils pourraient se montrer

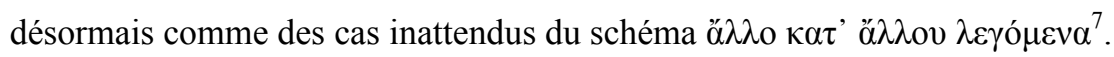

\footnotetext{
${ }^{4}$ F. Wolff, «Qu'est-ce que se demander ce qu'est la réalité ? ... », art. cit., p. 152, n. 16.

${ }^{5}$ J'emprunte le tour «faire substrat» à la traduction que F. Ildefonse et J. Lallot proposent pour le verbe טлокєі̃ $\theta \alpha$ dans leur traduction commentée de Catégories - Ildefonse Frédérique et Lallot Jean, Aristote, Catégories, texte grec - français avec introduction, notes et commentaire, Paris, éd. du Seuil, 2002. Ils justifient ce choix aux p. 154 et 302-303.

${ }^{6}$ D. Bostock (Aristotle. Metaphysics. Books ' and H, translation with a commentary, Oxford, Clarendon Press, 1994, p. 75): "But this is immensely puzzling, for it seems to be built into the matter/form analysis that form cannot be an ultimate subject of predication. on the contrary, form must surely be predicated of matter, and apparently of the compound too ». Tous les passages pertinents sont rapportés et analysés dans l'article classique de J. Brunschwig (1979), qui porte précisément le titre "La forme, prédicat de la matière? », dans Aubenque Pierre (éd.), Études sur la Métaphysique d'Aristote, Actes du vi ${ }^{\mathrm{e}}$ Symposium Aristotelicum, Paris, Vrin, 1979, p. 131-160. ; voir aussi Kung Joan, "Can Substance be Predicated of Matter? », Archiv für Geschichte der Philosophie, 60 (1978), p. 140-159.

7 Dans «Myles Burnyeat's Map of Metaphysics Zeta », The Philosophical Quarterly 55, ${ }^{\circ}$ 218, January 2005, p. 114-121[https://doi.org/10.1111/j.0031-8094.2005.00391.x], Mary Louise Gill s'est engagée résolument sur cette voie: «Reflection on matter in substantial generation reveals that composites in the first category resemble the accidental compound white man ». Si bien que «the analysis of substantial generation demonstrates that Categories-type primary substances, like Socrates and Bucephalus, plus their species and genera -man, horse, animal - fail to be primary in the Z's sense » (p. 119-120).
} 
Face à cet enjeu, Francis Wolff prend une position résolument concordiste et fait le pari d'une convergence «extensionnelle » des résultats sur lesquels débouchent les deux voies (apparemment parallèles) qui mènent à la découverte de l'ousia ${ }^{8}$. Il met en place à cet effet une hypothèse hardie : le choix des entités premières autour desquelles tournent aussi bien nos pratiques discursives que le devenir physique est gouverné, non pas par deux critères alternatifs (l'un visant le substrat, l'autre l'essence), mais par la double application d'un seul et même critère «schématique » (celui qui a été mis en place depuis les Catégories).

L'application physique de ce critère, lorsqu'elle est guidée (voire « rectifiée ») ${ }^{9}$ par les intuitions prédicatives ordinaires qui commandent la distinction entre substance et attribut, produit des résultats qui, loin de s'écarter de ces intuitions, les confirment. De ce point de vue, l'exercice de « déshabillage métaphysique $»^{10}$ auquel Aristote se livre dans Z, 3 tient beaucoup de la reductio ad absurdum : tout se passe comme s'il voulait montrer qu'une recherche unilatérale du fondement se solde par l'échec du discours prédicatif, car elle aboutit au «dépeuplement» de la première catégorie - celle habitée jusque-là par Socrate et Bucéphale. C'est le remplacement d'un sujet déterminé par un substrat résiduel qui parachève cette déchéance, car ce dernier « est si bien substrat premier dont on parle, qu'il n'est plus rien de dicible. La radicalité même du critère le perd $»^{11}$.

Mais l'application logique du critère doit être à son tour mise à l'épreuve du changement, pour déterminer si elle parvient à isoler non seulement ce qui, à un moment donné, tient lieu de sujet, mais aussi ce qui demeure dans ce sujet et permet de le reidentifier comme étant toujours le même. L'enjeu de l'identité diachronique est important parce que, tout comme le déshabillage de Z, 3, il risque de séparer le substrat physique du

\footnotetext{
${ }^{8}$ « Les deux lignes d'enquête [celle qui vise le substrat et celle qui vise la quiddité], correctement tracées, se croisent en ce point. Loin de diverger et de mener à des ousiai différentes, comme on le dit souvent (la matière d'un côté, la forme de l'autre), elles mènent à un résultat convergent » (Wolff, "Qu'est-ce que se demander ce qu'est la réalité ? ...», art. cit., p. 160).

${ }^{9}$ L'expression est de F. Wolff («Qu'est-ce que se demander ce qu'est la réalité ? ... », art. cit., p. 153).

${ }^{10} \mathrm{~F}$. Wolff («Qu'est-ce que se demander ce qu'est la réalité ? ... », art. cit., p. 158).

${ }^{11} \mathrm{~F}$. Wolff résume en ces termes la tournure paradoxale que prend cette démarche « radicale»: «C'est comme si la première catégorie (ce dont on parle et à propos de quoi on peut dire ce que c'est) était tellement catégorie première qu'on ne pourrait plus dire ce qu'elle est, répondre au 'qu'est-ce que ?' - ce qui est pourtant la définition de la première catégorie » ("Qu'est-ce que se demander ce qu'est la réalité ? ... », art. cit., p. 158). D'où la nécessité de « réinterpréter logiquement ce critère substrat pour qu'il s'accorde avec les exigences de la quiddité »(p. 161).
} 
sujet logique, c'est-à-dire le fondement du devenir de ce noyau qui en fait un sujet d'attribution identifiable sous une espèce. Et l'on sait bien que, aux yeux d'Aristote, rien ne saurait être sans être quelque chose: "il n'y aura de quiddité pour aucune espèce qui ne soit pas comprise sous un genre, mais seulement pour les espèces d'un genre » $(\mathrm{Z}, 4$, 1030a11-12) $)^{12}$.

Or ce parti pris concordiste engage Francis Wolff sur la voie d'une lecture très déterminée de ce pronom incertain qu'il repère à la ligne $1029 \mathrm{a} 2$ : il soutiendra que, en

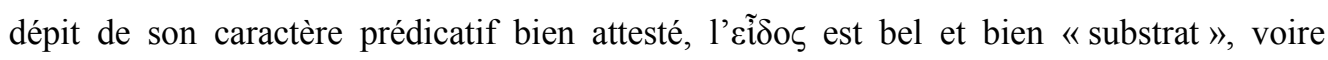
«substrat premier», et qu'il est à ce titre la réalité fondamentale dont tout le reste dépend. Pour le dire en un mot : le noyau de chaque chose est aussi son fondement ; non seulement la structure formelle qui la détermine à être ceci (plutôt que cela) ${ }^{13}$, mais aussi le véritable substrat des changements que cette chose sensible éprouve. Wolff écrit :

[S]ans doute, une certaine conception, historiquement première, du substrat premier mène à la matière, mais cette conception doit être rectifiée, car le vrai substrat premier, antérieur à la matière et au composé, c'est la forme. (Wolff, «Qu'est-ce que se demander ce qu'est la réalité ? ...», art. cit., p. 152-153) ${ }^{14}$

Aristote insistera d'ailleurs sur cette forme-substrat au premier chapitre du livre suivant qui revient sur les principaux acquis de la recherche menée dans le livre Z. Cette courte reprise offre pourtant une perspective nouvelle sur la portée de cette thèse, pertinemment mise en relief par David Bostock ${ }^{15}$. Bien que la manière la plus usuelle de «faire substrat» consiste à se donner comme une réalité à la fois déterminée et

\footnotetext{
${ }^{12}$ Selon les termes de Michael J. Loux, «There is no such thing as just existing : to exist is to instantiate a lowest-level essential predicate » (voir Loux M. J. Primary Ousia, An Essay on Aristotle's metaphysics Z and H, Cornell - New York, Cornell University Press, 1991, p. 73).

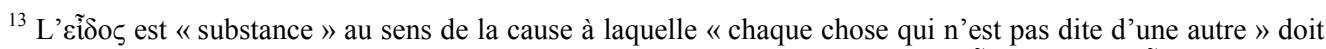

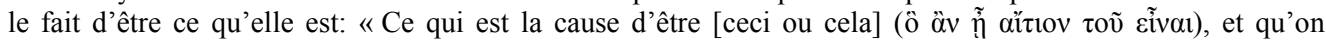
retrouve dans tout ce qui n'est pas énoncé d'un substrat, comme par exemple l'âme pour l'animal » (Metaph. $\Delta, 8,1017 \mathrm{~b} 14-16$, en prenant E⿱艹乂val, à la ligne $1017 \mathrm{~b} 15$, avec un attribut prédicatif sous-entendu. Cette lecture

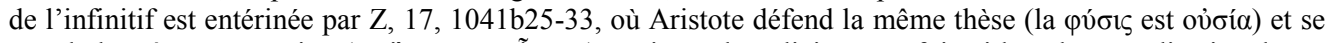

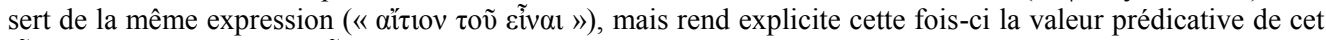

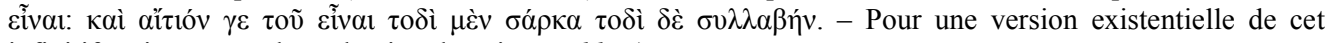
infinitif, voir pourtant la traduction de Tricot, ad loc.).

14 «[É]tant sujet logique et substrat physique, elle [sc. la forme] est à la fois antérieure logiquement a la matière et physiquement au composé » («Qu'est-ce que se demander ce qu'est la réalité ? ...», art. cit., p. 156, n.30).

${ }^{15}$ Cf. D. Bostock, Aristotle. Metaphysics. Books Z and H, op. cit., p. 251).
} 
déterminable (qua sujet d'attribution), Aristote préfère souligner ici le lien étroit qui existe

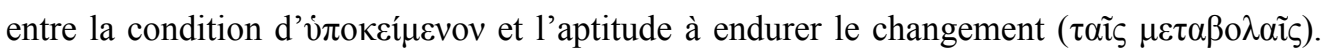
Ce changement prend, on le sait, la forme d'une alternance entre contraires, ce qui n'est pas sans rappeler le trait distinctif ( $\mu \alpha \dot{\lambda} \imath \_\tau \alpha$ íôıv) de l'ousia dans les Catégories, qui était précisément son aptitude à «recevoir les contraires tout en restant la même et numériquement une » $(4 \mathrm{a} 10-11)^{16}$. Est «substrat» tout ce qui demeure en tant que point d'attache fixe, auquel on peut accrocher ensuite des prédicats changeants $(c f . \mathrm{H}, 1$, 1041a32-b5).

Or, Francis Wolff semble croire que ces indications sur le rôle de substrat - axées sur la notion de permanence - peuvent être transférées mutatis mutandis au noyau formel du composé sensible, ce qui ouvre la voie à une façon de «sous-tendre » qui est enfin compatible avec l'idéalité d'une «forme ». Dans la mesure où elle assure la persistance et l'identité avec lui-même du composé qu'elle détermine (et qui est sujet du changement : ô

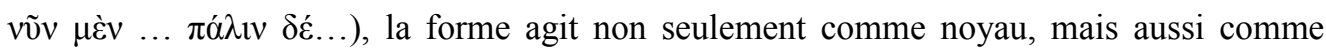
fondement : elle se montre comme « ce qui est un substrat dans ce qui est un substrat» (Wolff $^{17}$. Cela ne lui accorde pas, bien entendu, une priorité absolue et sans restriction dans tous les domaines de l'être et selon tous les critères: Francis Wolff prend soin de préciser que la forme, aussi bien que la matière et le composé, « ont tous les trois des titres à être la réalité des réalités sensibles parce qu'ils ont tous les trois des titres de priorité par rapport aux deux autres » («Qu'est-ce que se demander ce qu'est la réalité ? ... », art. cit., p. 155). Ainsi, il n'y a que le composé sensible qui soit «séparable» sans aucune restriction et sous tous les rapports ( $\dot{\alpha} \pi \lambda \tilde{\omega} \varsigma, H, 1,1042 \mathrm{a} 30-31)$; on ne peut pas en dire autant ni de sa matière ni de sa forme. L'application physique unilatérale du critère-substrat dévoile un fondement qui sous-tend le devenir et qui est premier dans ce domaine; mais une telle primauté ne s'étend pas au discours attributif, car ce fondement restera anonyme et indicible. De son côté, une application purement logique du même critère parvient à identifier les points d'attache du discours attributif, mais reste aveugle à leur complexion prédicative interne. Par contre, une application nuancée et coordonnée des deux approches

\footnotetext{
${ }^{16}$ Je cite d'après la traduction de Crubellier, Dalimier et Pellegrin (Michel Crubellier, Catherine Dalimier et Pierre Pellegrin, Aristote. Catégories, Sur l'interprétation, Paris, GF Flammarion, 2007, p. 125).

${ }^{17}$ «Qu'est-ce que se demander ce qu'est la réalité ? ... », art. cit., p. 160.
} 
produira précisément la conséquence qu'on s'accorde à reconnaître comme le résultat capital du livre $\mathrm{Z}$ : la primauté de la forme aussi bien sur la matière que sur le composé (annoncée déjà en Z, 3, 1028b29-33; cf. 1028a31-33) :

La forme est substrat physique du composé et substrat logique de la matière, et c'est en ce sens qu'elle est substrat premier, c'est-à-dire, antérieur à l'un et à l'autre. (Wolff, «Qu'estce que se demander ce qu'est la réalité ? ...», art. cit., p. 155).

Dans ce qui suit, nous chercherons à apporter quelques évidences textuelles en faveur de cette formulation abrégée de Francis Wolff autour du double rôle fondamental qu'il fait jouer aux formes dans l'économie du livre Z. Ce faisant, on se donnera les moyens de confirmer son interprétation de 1029a2, qui fait du noyau formel d'un composé sensible son

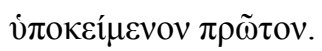

\section{L'eĩios, " substrat physique du composé »}

Que la forme d'une chose puisse lui tenir lieu de substrat est une éventualité qu'Aristote prend très au sérieux dans De generatione et corruptione I, 5. À cet endroit il s'aperçoit qu'on ne peut pas toujours compter sur la matière du composé pour assurer ce rôle : son insuffisance est rendue manifeste dès qu'on s'interroge sur le véritable sujet d'un accroissement ou d'une prise de poids. Lorsqu'un animal croît, qu'est-ce qui subit cette augmentation : sa matière ou sa forme ? On serait tenté de répondre, de prime abord, que sa matière, puisque c'est le corps de l'animal qui éprouve un changement et non pas son âme, laquelle reste inchangée (cf. DA I, 3, 406a2-4 ; Phys. VIII, 5, 258a18-21). On sait bien, en outre, que ce corps qui grossit est souvent identifié par Aristote à la matière des vivants : đò $\delta \dot{\varepsilon} \sigma \tilde{\omega} \mu \alpha$ ṽ$\eta \eta(\mathrm{Z}, 11,1037 \mathrm{a} 6)^{18}$. Mais, à y regarder de plus près, on s'aperçoit que cette réponse initiale (envisagée et rejetée dans $G C$ I, 5, 321a16-17) est inexacte. Aristote pose la question du sujet d'un accroissement à l'échelle des membres locomoteurs : si une jambe devient plus grande, il semble plausible qu'elle le fasse par l'annexion successive de nouvelles portions de chair et d'os ( $c f .321 \mathrm{a} 30-34$; b19-25), que la nourriture assimilée contenait « en puissance » (322a5-7).

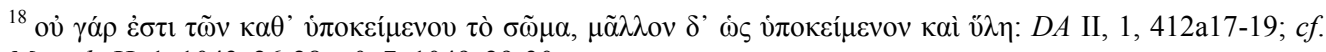
Metaph. H, 1, 1043a26-28; $\theta$, 7, 1049a29-30. 
Aristote a pris soin d'établir auparavant une exigence qui semble aller de soi, mais qui n'est pas superflue dans ce contexte : pour qu'il y ait de la croissance, il faut pouvoir compter sur un substrat sous-jacent qui demeure et dont on pourra dire, au bout du

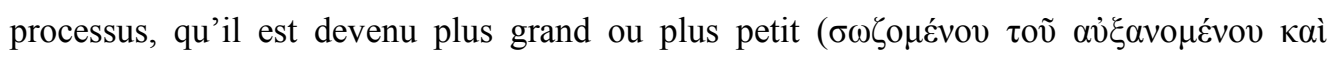

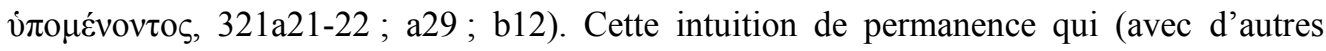
axiomes apparentés) commande l'analyse, laisse à découvert un substrat passablement inattendu. En effet, l'assimilation de nourriture par un individu auto-identique suggère que ce qui demeure chez lui n'est pas sa matière (à chaque fois renouvelée par le remplacement continu de ses parties homéomères : 321b18), mais sa forme. Pour le montrer, Aristote introduit ici sa thèse de la double référence des expressions mixtes, c'est-à-dire, de celles qui renvoient à « une chose dans une autre » $(\tau \text { ó } \delta \varepsilon \dot{\varepsilon} v \tau \tilde{\omega} \delta \varepsilon)^{19}$ ou à des choses dont la forme

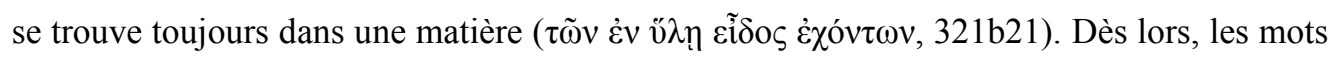
« chair » et « os » peuvent désigner tour à tour la forme ou la matière des réalités doubles auxquelles ils s'appliquent.

Or la chair qua matière ne demeure pas et peut être comparée à la section (arbitrairement définie) d'un fleuve, qui n'est jamais remplie par une portion

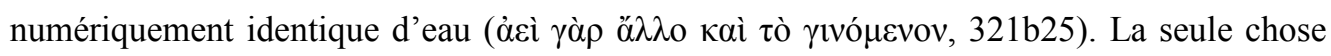
qui demeure au milieu de cet écoulement, c'est la mesure imposée aux eaux toujours changeantes: cette structure permettant de repérer une section du fleuve en dépit de l'absence des eaux qui la remplissent à un moment donné. La délimitation reste la même, bien que les eaux délimitées changent sans cesse. Il faut donc penser aux parties organiques

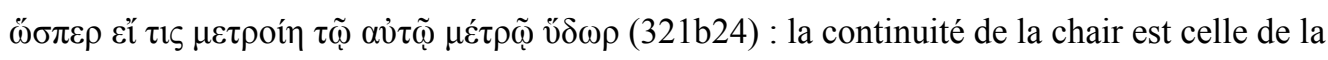

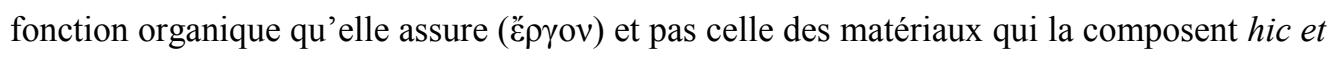
nunc. L'intuition selon laquelle c'est le corps qui croît doit être donc nuancée : c'est en tant

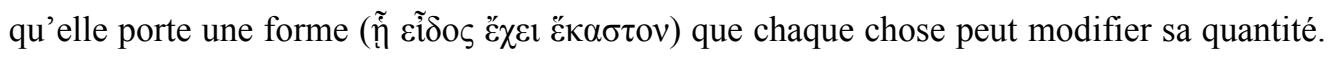
Sa matière ne croît pas parce que (au bout d'un moment) elle n'est plus là pour éprouver quoi que ce soit ${ }^{20}$. Ce point a été tiré au clair par G.E.M. Anscombe ${ }^{21}$ :

\footnotetext{
${ }^{19}$ Metaph. Z, 5, 1030b17-18 ; Z,11, 1036b21-25 ; cf. Z, 8 Z 8, 1034a5-7.

${ }^{20}$ « Nutrition is not simply adding new material to already existing organs, but continually remaking organs that are in continual decay - for Aristotle's picture of the living body, described especially in the De juventute (and cf. GC 1.5, 321b21-8), is starkly Heraclitean »: S. Menn, "Aristotle's definition of soul and the programme of the De Anima », Oxford Studies in Ancient Philosophy 22, 2002, p. 83-139, (ici p. 122).
} 
[M]atter can be added or taken away, but cannot be said to grow, for growth is by addition of matter. Thus it is that we use the term designating the kind of thing, to stand for the subject of growth.

D'où il s'ensuit qu'on n'a pas le droit d'affirmer que «the identity of a person is the identity of the matter of which he is composed ». Francis Wolff songe peut-être à ce genre de continuité fonctionnelle lorsqu'il fait de l'eĩ̇o $\delta$ le « substrat physique du composé ».

\section{L'eĩ்os, « substrat logique de la matière »}

Au moins à deux reprises au cours du livre Z, Aristote affirme la subordination logique de la matière à la forme. La première mention s'inscrit dans une remarque annexe placée au cœur de la «méréologie définitionnelle» du chapitre 10, où Aristote est concerné par l'exclusion du bronze de (la formule qui exprime) la quiddité d'une statue :

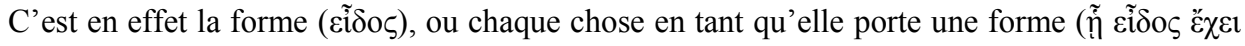

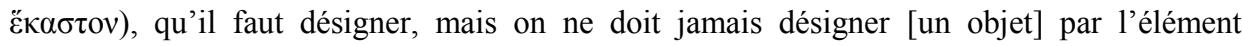
matériel pris en lui-même. (Z, 10, 1035a7-9, traduction Tricot modifiée).

L'observation revient dans un passage du chapitre suivant où il est question de l'instabilité que la composition matérielle introduit dans les objets de la connaissance, ce qui rend difficile leur appréhension in absentia - si ce n'est par recours à l'universel qu'ils incarnent :

Or pour ce qui est du composé, tel que ce cercle-ci et n'importe quel autre des cercles individuels, qu'il soit sensible ou intelligible [...], de ces composés-là, dis-je, il n’y a pas de définition, mais ils sont connus avec l'intellect ou la sensation; et s'ils manquent l'actualisation, il n'est pas clair s'ils existent ou non. Mais ces choses-là sont toujours énoncées et connues grâce à leur énoncé universel. La matière, en revanche, reste

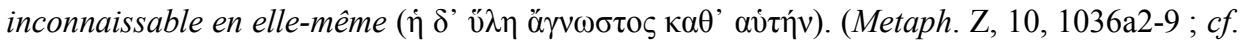
$\mathrm{Z}, 15,1039 \mathrm{~b} 27-30)$

Ce qui semble certain, c'est que la présence du matériau rend imprécis et indéfini l'énoncé d'un composé que seule sa forme peut rendre clair : sous peine d'obscurité, il faut

${ }^{21}$ Anscombe Gertrude Elizabeth Margaret, « The principle of individuation. II. », dans G.E.M. Anscombe, The Collected Papers of G.E.M. Anscombe I: From Parmenides to Wittgenstein, Oxford, Blackwell, 1981, p. $57-65$; ici p. 65 . 


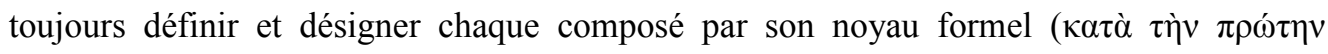

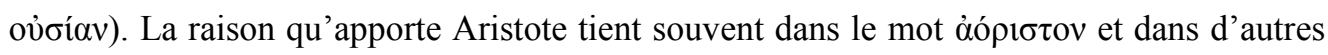
termes apparentés ${ }^{22}$. La présence de ces qualifications privatives a pu suggérer qu'il vise avant tout la materia prima, en tant qu'ingrédient qui peut être omis (ou incorporé) dans l'énoncé d'un composé sensible ${ }^{23}$. Certes, la connexion se suggère d'elle-même, en raison de l'obscurité de ce substrat résiduel obtenu par Aristote au moyen d'un «déshabillage métaphysique »; il nous semble, cependant, qu'il s'agit là d'un choix interprétatif «par défaut 》, motivé surtout par la difficulté à attacher un sens précis aux adjectifs «indéfini » et «inconnu», lorsqu'ils sont apposés aux matériaux familiers qui rentrent dans la constitution des choses sensibles. En même temps, ce sont ces matériaux-là qui semblent mieux placés pour faire partie du definiens de la chose qu'ils constituent, au lieu d'un substrat premier dont on ne connaît pas le nom.

On peut trouver un usage parallèle de ces notions privatives, au service d'une stratégie analogue de subordination logique, dans le traitement de la notion d'« infini » (ou

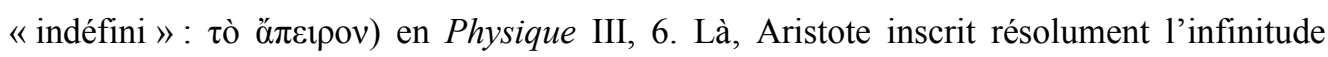
dans les limites d'un corps sensible et pense l'illimité à partir des limites qui le rendent concevable, ce qui a pour conséquence une réorientation radicale du concept par rapport à l'héritage présocratique. Tout comme la matière de Z, 10-11, l'in(dé)fini figure ici comme

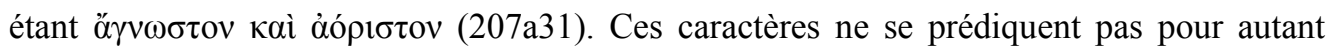
d'un substrat résiduel qui serait hors de portée pour un discours prédicatif toujours axé autour d'un sujet identifiable. L'idée (passablement présocratique) d'une étendue illimitée qui «contient» et «gouverne » toute $\operatorname{chose}^{24}$, cède le pas à celle d'une extension bien cernée, quoique divisible sans terme. De «continent universel» qu'il était (notamment, chez Anaximandre), l'infini devient, de ce fait, un contenu bien délimité. Comme le dit

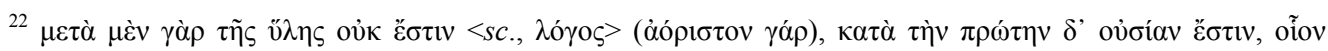

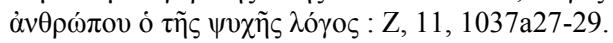

${ }^{23}$ C'est l'avis de W. D. Ross (Arisotle, Metaphysics, A revised Text with Introduction and Commentary, Oxford, Clarendon Press, 1924 : p. 205, ad 1037a27) et, plus récemment, de M. Bastit (2002). Après lui avoir décerné le titre de « réalité ", Bastit dit de la matière concernée qu' " elle n'est pas rien mais elle est pure puissance »; ce qui lui permettrait de «cohabiter avec la forme substance première dans le composé. Elle s'intègre ainsi par exemple à sa définition » (Bastit Michel, Les quatre causes de l'être selon la philosophie première d'Aristote, Louvain-La-Neuve - Paris, Sterling - Virginia, 2002, p. 240). Concernant le sujet

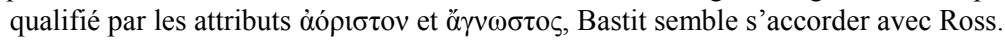

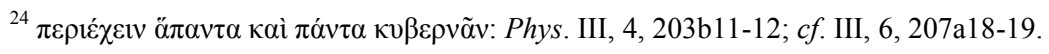


Aristote lui-même dans une formule qui prend le contre-pied d'Anaximandre : кaì oủ

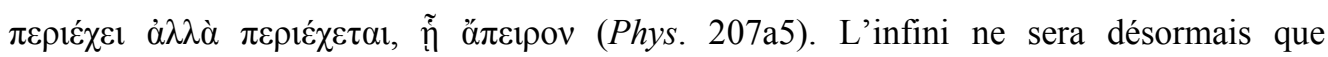
l'aptitude qu'une extension matérielle révèle lorsqu'elle est soumise à des actes successifs de division, qui peuvent être itérés sans terme. Dans le cadre du traitement aristotélicien de l'infini, les deux attributs privatifs de Métaphysique Z,10-11 sont donc rattachés, non pas à un substrat universel qui contiendrait tout en son sein, mais à celui qui est, tour à tour, « contenu » par les limites d'une chose concrète. C'est cette matière faisant partie ( $\mu$ óptov) d'un composé qui se voit maintenant décerner les titres d'inconnue et d'indéfinie ( $\tau$ ò

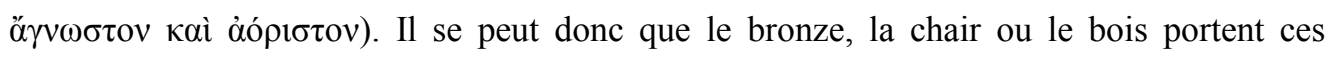
qualifications à peu près dans le même sens.

Ces deux opérations de subordination conceptuelle (autant celle accomplie dans Physique III, 6 que celle à l'œuvre dans Metaph. Z, 10-11) attestent, chacune à sa façon, la dépendance logique de la matière par rapport à la forme qui définit chaque chose, si bien que le seul illimité est celui que l'on découvre à l'intérieur d'un corps sensible. On peut en dire autant du substrat, ce qui apporterait un soutien indirect à la lecture de Z, 3 comme une reductio ad absurdum, plutôt que comme l'introduction d'une entité métaphysique sui generis.

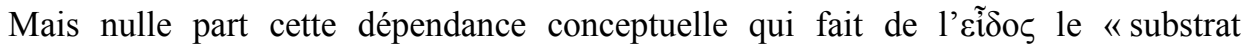
logique » de la matière n'est plus sensible que dans les développements d'Aristote autour des appellations matérielles d'une chose ; c'est-à-dire de ces appellations qu'on lui impose à partir des matériaux dont elle est faite. Dans ce traitement, la matière d'un objet apparaît comme un attribut parmi d'autres, attribut que son appartenance même à la substance rend déterminé. Loin de figurer comme un item indépendant, sur lequel l'ontologie porterait un éclairage privilégié, la matière n'est rendue intelligible qu'à partir de la chose qu'elle constitue, et doit être saisie à partir de celle-ci. Ce faisant, Aristote renverse l'ordre de priorité établi par les anciens naturalistes, aux yeux de qui les individus sensibles se détachaient sur le fond d'un substrat homogène, en tant que «affections, états ou

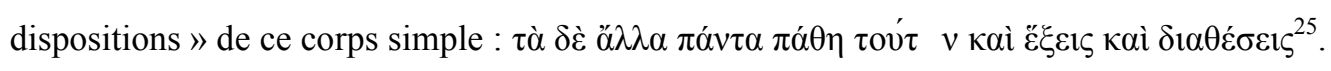

${ }^{25}$ Phys. II, 1, 193a25. Le pronom relatif $\tau$ ov́ $\omega$ v renvoie ici au corps simple que chaque «physiologue » identifie comme substrat universel. 
En revanche, pour Aristote ce n'est pas l'objet qui doit être conçu comme une affection de sa matière, mais plutôt la matière qui devrait être prise pour une affection de l'objet.

Ce parti pris est particulièrement net dans les distances que prend Aristote par rapport au Timée en ce qui concerne l'attachement des quatre corps simples à leur substrat immanent. Tel qu'Aristote reconstruit son propos, Platon aurait minimisé la portée des différences formelles entre les éléments, au profit d'un substrat qui demeure dans le changement. Par ce biais, Platon favorisa la dénomination de chaque entité à partir de la matière dont elle est faite, sans remarquer que l'vं devenir seulement dans les cas d'altération. En effet, dès qu'il est question de l'avènement d'une substance, tout ce qui demeure dans le terminus ad quem d'un processus ne constitue qu'une affection $\left(\pi \alpha \dot{\theta} \theta \varsigma_{\varsigma}\right)$ du produit de la genèse. C'est ce qui arrive, par exemple, lorsque l'air est changé en eau, et que le produit garde la transparence et la froideur de son point de départ (319b22-24) ; c'est ce qui arrive aussi, ex hypothesi, lorsque l'or d'une couronne est réutilisé dans la fabrication d'un tuyau de plomberie : là, le produit n'est pas dénommé par son point de départ - «ceci est de l'or». Au contraire, ce qui demeure au cours du changement est désigné, désormais, par son inscription dans le produit engendré (« ce tuyau est en or »). De sorte que le substrat y apparait comme logiquement «parasitaire» du produit achevé. Dans le De generatione et corruptione, Aristote avance un critère tranchant, permettant de décider si un processus quelconque est une génération ou une altération et, par là même, si son résultat peut être désigné sans réserves à partir de son

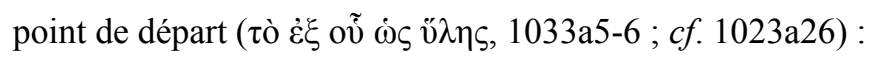

Si une certaine affection relevant d'une contrariété demeure la même aussi bien dans ce qui est engendré que dans ce qui se corrompt [...] il ne faut pas que le terminus ad quem du

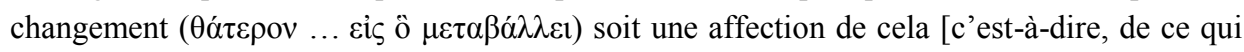

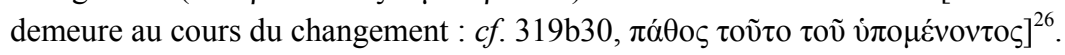

Sous peine de suggérer, à tort, que l'essentiel d'une chose réside dans son substrat, la référence à ce dernier doit emprunter le détour de l'objet engendré, de façon à attester sa

\footnotetext{
${ }^{26}$ GC I, 4, 319b21-24. Une version raccourcie du même critère apparaît dans 319b33. W. Charlton, Aristotle's Physics Book I and II, translated with Introduction and Notes, Oxford, Clarendon Press, 1970, p. 75) commente : "That is, if the terminus ad quem is parasitic on, called real because of its relation to, anything which remains throughout the change, the change is an alteration; if whatever remains throughout the change is parasitic on the terminus ad quem, the change is a coming into existence ».
} 
dépendance logique à l'égard du produit : ce dernier n'est pas bois, mais «de bois », et ainsi de suite. Si le point de départ matériel - le « $\dot{\varepsilon} \xi$ oṽ $\omega \varsigma$ vँ $\lambda \eta \varsigma »^{27}$ - fournit encore une appellation valable du $\gamma(\gamma v$ ó $\mu \varepsilon v o v$, il faut rendre claire sa position subordonnée au moyen d'une modification flexionnelle imposée au nom du matériau. Aux yeux d'Aristote, le manque de cette paronymie dans le Timée trahit un malentendu fondamental, qui identifie le noyau de chaque chose à son fondement :

[P]our les choses dont il y a génération ou corruption, il est impossible d'appeler le produit

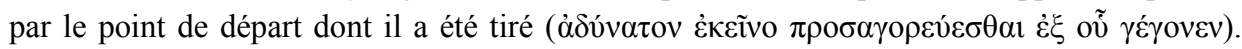
Pourtant, [Platon] affirme qu'il est vrai au plus haut point de dire de chacun des produits en

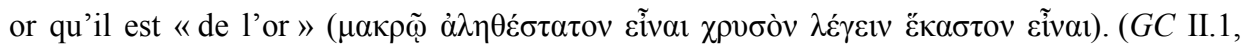
$329 \mathrm{a} 19-21)^{28}$

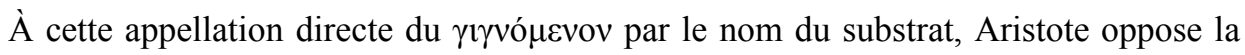

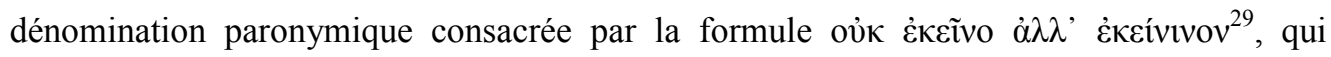
parachève à peu près l'opération inverse. Aristote sous-entend par là que les conditions d'identité d'un objet quelconque ne sont pas fixées par l'étoffe dont il est fait, de sorte qu'on ne peut pas l'appeler sans plus par le nom de sa matière. Il faudrait, au contraire, définir le matériau, qua matériau, en fonction de l'objet qu'il constitue. Attendu que plusieurs choses peuvent avoir en commun le même type de matière sans détenir, de ce seul fait, les mêmes pouvoirs, la primauté explicative reviendra à leur forme. C'est la priorité logique de la forme qui recommande désormais d'en tirer seulement une appellation oblique.

Un indice décisif en faveur de cette priorité est apporté par le rigoureux parallélisme grammatical qu'Aristote détecte entre deux registres différents de la langue. Dans Métaphysique $\Theta, 7$, il indique que le «langage attributif» (qui lie les noms des attributs aux objets qu'ils qualifient) suit le même patron de dérivation flexionnelle imposée

\footnotetext{
${ }^{27}$ Metaph. Z, 7, 1033a5-6.

${ }^{28}$ Pour l'accusation d'Aristote, selon laquelle Platon choisirait le nom du matériau comme l'appellation la plus juste convenant à chacun des objets en or, voir Timée 50a-b. A. Code («The persistence of Aristotelian matter », Philosophical Studies: An International Journal for Philosophy in the Analytic Tradition, Vol. 29, No. 6 (Jun., 1976), p. 357-367, repris dans Lloyd Gerson P. (éd.), Aristotle. Critical Assessments, Volume II: Physics, Cosmology and Biology, London - New York, Routledge, 1999, p. 25-34 - ici p. 34) voit dans GC II, 1 «(i) a statement that Plato talked about gold products, but that 'this is not well spoken being said in such a manner', and then (ii) a sketch of the explanation given at Met. Z, 7 for the impropriety of such locutions ».

${ }^{29}$ Metaph. Z, 7, 1033a6-7. Cf. Metaph. Z, 7, 1033a5-19.
} 
auparavant au « langage constitutif » (qui relie les matériaux aux choses qu'ils constituent). Après avoir repris le point familier de Z, 7 concernant la constitution des composés

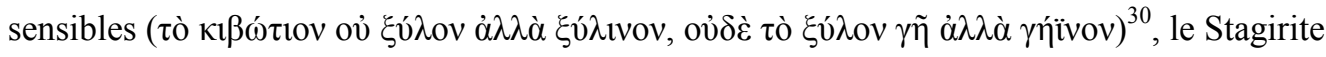
remarque que les qualités et autres affections reçoivent le même traitement grammatical :

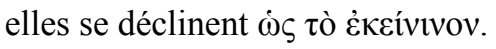

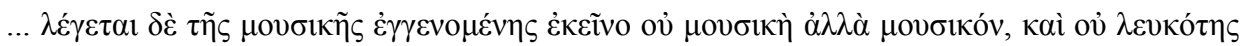

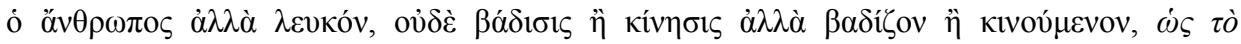
ÉKeivivov ${ }^{31}$.

Sur ce point Aristote n'est pas seul. Son élaboration rejoint de près le traitement conjoint des termes de masse et des termes qualitatifs avancé par Peter Strawson ${ }^{32}$, qui a reconnu dans ces deux catégories d'expression des universaux authentiques («true generalthing names »), à la différence des noms de substance, lesquels font double emploi. Pour ce qui est de ces derniers, la désignation indéfinie de l'individu concret - « un cheval » - tient souvent lieu d'universel, à défaut d'une expression abstraite destinée à ce seul effet. On n'éprouve pas d'ordinaire l'urgence de réserver un nom à la mention exclusive $d u$ caractère universel, sauf dans certains contextes théoriques restreints, où les philosophes se sont donné la peine d'échafauder des abstractions telles que «chevalinité » ou «humanité ${ }^{33}$. Mais ces noms ne font pas partie du lexique habituel ; leur introduction occasionnelle est motivée par le projet de rendre homogène l'exemplification de ces prédicats avec celle des qualités abstraites authentiques. Sur ce point, ce que Strawson appelle les véritables noms d'universel (i.e., les termes de masse et les noms de propriétés) vont sur le même bateau sémantique, car tous les deux diffèrent des noms attachés aux substances :

\footnotetext{
${ }^{30}$ Met. $\Theta, 7,1049$ a19-20 : «Lorsque la musique vient à se trouver dans un sujet donné, on n'appelle pas ce dernier "musique", mais "musicien", on ne dit pas que l'homme est "blancheur", mais "blanc", ou qu'il soit "mouvement"' ou "marche", mais qu'il est "en marche" ou "en mouvement", comme on disait "de cela" [lorsqu'il était question du rapport entre les choses et de leur matériau] » (trad. Tricot modifiée).

${ }^{31}$ Met. $\Theta, 7,1049 \mathrm{a} 30-4$.

32 «Particular and general » (1954).

${ }^{33}$ Cf. P. Strawson («Particular and general », dans Proceedings of the Aristotelian Society, Volume 54, Issue 1, 1 June 1954, p. 233-260, ici p. 240).
} 
Given a true general-thing name, like "gold" or "wisdom", the question of the criteria of identity of its instances cannot be answered until the kind of instance is specified, by such a phrase as "a piece of gold" or " $a$ wise action",34.

Nous voudrions suggérer ici que la conscience de ce régime sémantique commune aux matériaux et aux affections amène Aristote à regrouper les termes de masse et les noms de qualités sous le même patron flexionnel, et à concevoir autant les matériaux que les affections comme «indéfinis » par rapport à leurs instances. Cette valeur attachée au

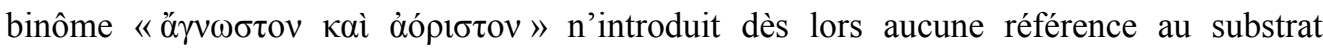
résiduel de Z, 3 dans le definiens d'un composé, puisque ces expressions privatives sont compatibles avec les matériaux de tous les jours. Indépendamment des mérites philosophiques de l'analyse de Strawson ${ }^{35}$, ce qu'il importe de souligner ici est avant tout la convergence sémantique de ces deux catégories d'expression qu'Aristote a placé du côté de

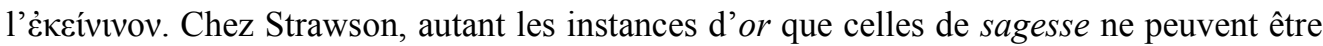
précisées qu'in obliquo, à l'aide d'une expression nominale à laquelle ils s'attachent en qualité d'épithètes. C'est cette dépendance qui les rend déterminées. Si l'on tient compte de cette structure parasitaire commune aux deux classes de prédicat, on peut rendre lisible le parallèle quelque peu déroutant qu'Aristote établit, dans les dernières lignes de Métaphysique $\Theta, 7$, entre la matière d'une chose concrète et toutes ses autres qualités. Là il est dit qu'une substance est qualifiée à la fois par ses affections et par sa matière ; si bien qu'il est raisonnable qu'elle reçoive une appellation paronymique tirée du nom de chacun de ces aspects qu'on y découvre :

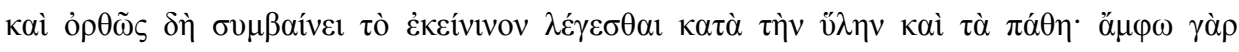

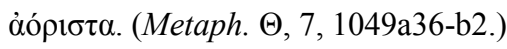

Aristote attire ainsi l'attention sur le fait que, dans plusieurs contextes, les références

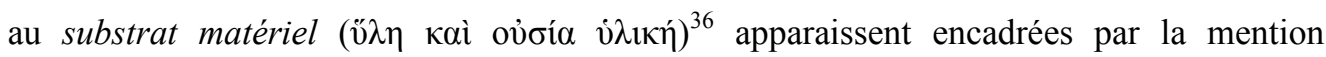

\footnotetext{
${ }^{34}$ P. Strawson («Particular and general », art. cit., p. 255). Le passage se poursuit par un contraste: «But where one set of criteria of identity is peculiarly dominant, its adoption peculiarly compelling, we find no such non-committal general name in current, adult, unsophisticated use. All that we might wish to do with it, we can equally well do without it, by the use of the indefinite singular or plural forms of the ordinary substance-name (e.g., "a horse” or "horses"). "

${ }^{35}$ Pour quelques réserves, $c f$. Cartwright, H. M. (1970).

${ }^{36}$ Met. $\Theta, 7,1049 \mathrm{a} 36$.
} 


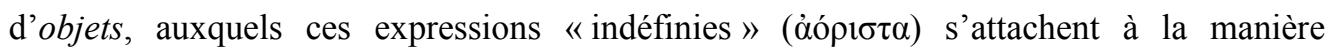
d'attributs. Et il s'aperçoit qu'un phénomène analogue se produit avec d'autres catégories d'expression, qui possèdent à peu près la même dépendance logique ( $\tau \dot{\alpha} \pi \alpha \dot{\alpha} \theta \eta$ ). On ne dénombre pas, en effet, les instances individuelles du courage sans les faire tomber d'abord sous un autre concept, permettant de les compter. Ainsi, il n'y a aucun individu qui soit courage, justice ou couleur; ces qualités sont exemplifiées, selon les cas, par des courageux, des justes ou des colorés, ce qui présuppose l'identification préalable du porteur de qualités sous un autre concept, avec la modification flexionnelle du nom abstrait qui s'ensuit.

La présence d'une paronymie analogue touchant les noms des matériaux n'est pas, au premier abord, une coïncidence. Elle s'explique par le fait que, autant ces noms que ceux des attributs, se trouvent placés sous la même dépendance. Aristote anticipe ainsi l'intuition strawsonienne d'après laquelle tant le mot « rouge » que le mot «bois » manquent d'un critère permettant d'individualiser leurs instances. Ne portant pas en eux-mêmes leur propre arithmétique, leur exemplification passe souvent par celle d'un autre terme, seul moyen

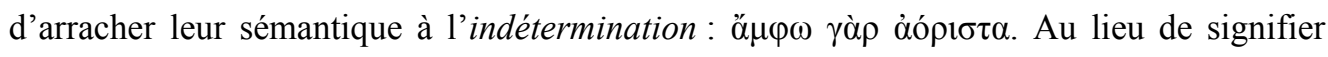
une substance à part entière, ces termes ont tendance à désigner un moment constitutif, ou un aspect qualitatif, d'une réalité proprement dite.

Francis Wolff rend justice à ces intuitions de dépendance logique lorsqu'il appelle à ne plus penser au substrat comme à « une réalité autonome » (Wolff, "Qu'est-ce que se demander ce qu'est la réalité ? ...», art. cit., p. 157), et à reformuler, si nécessaire, la liste des quatre candidats au titre de ousia avancée au début de Z, 3. Wolff observe, en effet, qu'il y a une asymétrie remarquable dans cet inventaire initial, puisque trois de ses quatre

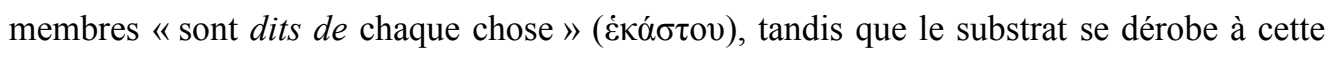
structure ousia + génitif (Wolff, «Qu'est-ce que se demander ce qu'est la réalité ?... », art. cit., p. 149). Or des analyses précédentes autour de la dépendance logique de la matière à l'égard d'une forme qui la détermine s'ensuit le réalignement du critère-substrat sur la structure prédicative commune aux trois autres candidats. Une telle démarche, consistant à 
recadrer le substrat dans sa référence au composé, est ce que Wolff appelle la « réinterprétation logique » de ce critère et constitue l'un des acquis centraux du livre Z. En particulier, l'introduction des vivants comme les véritables individus (Z, 10-11 et Z, 16) infléchit la recherche du fondement dans une nouvelle direction :

Le «substrat» sera bien, des lors, le substrat de chaque particulier; il pourra être mis du même côté que les trois autres critères, le bon (la "quiddité») comme les deux mauvais (l'universel et le genre), et être pour ainsi dire construit comme eux avec le génitif hekastou, comme au début de l'enquête (1028a34-36) : la réalité n'est pas en effet la quiddité de chacun ou le substrat ultime, elle n'est pas non plus la quiddité de chacun et le substrat ultime, elle est ultimement la quiddité et le substrat de chacun. (F. Wolff, «Qu'est-ce que se demander ce qu'est la réalité ?...», art. cit., p. 161).

Il est difficile de mieux exprimer cette stratégie de convergence, qui permet au noyau de chaque chose de rejoindre enfin son fondement.

\section{Bibliographie}

Anscombe Gertrude Elizabeth Margaret, «The principle of individuation. II. », dans G.E.M. Anscombe, The Collected Papers of G.E.M. Anscombe I: From Parmenides to Wittgenstein, Oxford, Blackwell, 1981, p. 57-65. [1 $1^{\mathrm{e}}$ éd. : "Symposium: The Principle of Individuation », Proceedings of the Aristotelian Society, Supplementary Volumes, Vol. 27, Berkeley and Modern Problems (1953), p. 83-96].

Aubenque Pierre (éd.), Études sur la Métaphysique d'Aristote, Actes du $\mathrm{VI}^{\mathrm{e}}$ Symposium Aristotelicum, Paris, Vrin, 1979.

Bastit Michel, Les quatre causes de l'être selon la philosophie première d'Aristote, Louvain-LaNeuve - Paris, Sterling - Virginia, 2002.

Bostock David, Aristotle. Metaphysics, Books Z and H, translation with a commentary, Oxford, Clarendon Press, 1994.

Brunschwig Jacques, «La forme, prédicat de la matière? », dans Aubenque Pierre (éd.), Études sur la Métaphysique d'Aristote, Actes du VI ${ }^{\mathrm{e}}$ Symposium Aristotelicum, Paris, Vrin, 1979, p. 131-160.

Cartwright Helen Morris, «Quantities », Philosophical Review 79 (1970), p. 25-42.

Code Alan, "The persistence of Aristotelian matter», Philosophical Studies: An International Journal for Philosophy in the Analytic Tradition, Vol. 29, No. 6 (Jun., 1976), p. 357-367, repris dans Lloyd Gerson P. (éd.), Aristotle. Critical Assessments, Volume II: Physics, Cosmology and Biology, London - New York, Routledge, 1999, p. 25-34.

Charlton William, Aristotle's Physics Book I and II, translated with Introduction and Notes, Oxford, Clarendon Press, 1970. 
Crubellier Michel, Catherine Dalimier et Pierre Pellegrin, Aristote. Catégories, Sur l'interprétation, Paris, Flammarion GF, 2007.

Lloyd Gerson P. (éd.), Aristotle. Critical Assessments, Volume II: Physics, Cosmology and Biology, London - New York, Routledge, 1999.

Gill Mary Louise, « Myles Burnyeat's Map of Metaphysics Zeta », The Philosophical Quarterly 55, $\mathrm{n}^{\circ} 218$, January 2005, p. 114-121. https://doi.org/10.1111/j.0031-8094.2005.00391.x

Ildefonse Frédérique et Lallot Jean, Aristote, Catégories, texte grec - français avec introduction, notes et commentaire. Paris, éd. du Seuil, 2002.

Kung Joan, « Can Substance be Predicated of Matter? », Archiv für Geschichte der Philosophie, 60 (1978), p. 140-159.

Loux Michael J., Primary Ousia, An Essay on Aristotle's metaphysics Z and H, Cornell - New York, Cornell University Press, 1991.

Menn Stephen, "Aristotle's definition of soul and the programme of the De Anima », Oxford Studies in Ancient Philosophy 22, 2002, p. 83-139.

Strawson Peter Frederick, «Particular and general », dans Proceedings of the Aristotelian Society, Volume 54, Issue 1, 1 June 1954, p. 233-260, https://doi.org/10.1093/aristotelian/54.1.233

Wolff Francis, «Qu'est-ce que se demander ce qu'est la réalité ? », dans M. Narcy et A. Tordesillas (éd.), La "Métaphysique» d'Aristote, Perspectives Contemporaines, Paris - Bruxelles, Vrin Ousia, 2005, p. 145-169. 